\title{
The role of transition-state bond-order on structure-reactivity relationships: the case of pericyclic and pseudo-pericyclic reactions
}

\author{
Paulo E. Abreu, Alberto A. C. C. Pais, and Sebastião J. Formosinho* \\ Chemistry Department, University of Coimbra \\ Rua Larga, 3004-535 Coimbra, Portugal \\ E-mail: sformosinho@qui.uc.pt
}

\begin{abstract}
This work is dedicated to Prof. António Rocha Gonsalves, on the occasion of his $70^{\text {th }}$ birthday: to the colleague and to the scientist who contributed to the development of modern organic chemistry in Coimbra
\end{abstract}

\begin{abstract}
The importance of the transition-state bond-order $\left(n^{*}\right)$ in the interpretation of chemical reactivity, in complement to the effect of reaction energy, for decarbonylation pericyclic and pseudopericyclic reactions of 3-cyclopentenone and related molecules is demonstrated through the intersecting-state model and quantum-mechanical electronic structure calculations. Both types of methodology reveal that $n^{\dagger}$ is strongly related to transition state geometry.
\end{abstract}

Keywords: Pericyclic reactions; structure-reactivity relationships; intersecting-state model (ISM); transition-state bond-order

\section{General Aspects}

Based on the idea that the ability of molecules to react rests ultimately on their own structural properties, chemists have employed several types of mathematical equations to express the relation of molecular-structure to reaction rate-constants or energy barriers. However such approaches do not currently encompass the role of electronic factors. This neglected factor often counters the energetic effects and gives rise to 'anomalies' or separate correlations. Recently Arnaut and Formosinho presented a quantification of the concept of 'chemical reactivity', emphasizing the role of molecular and electronic factors in chemistry through the Intersecting (interacting)-state model (ISM). ${ }^{1}$

ISM is based on simple chemical concepts: chemical bonds, represented in terms of potential energy curves (e.g. force constants for reactants, $f_{\mathrm{r}}$, and products, $\left.f_{\mathrm{p}}\right)$ and bond lengths $\left(l_{\mathrm{r}}\right.$ and $\left.l_{\mathrm{p}}\right)$, reaction energy, and bond orders for chemical bonds in the transition states (transition-state bond-order, $n^{\ddagger}$ ). The model incorporates novel insights in order to understand chemical reactivity for bond-breaking-bond-forming processes. ${ }^{2}$ First, the electronic element: the encounter of two molecules triggers unique features which neither molecule possesses alone, notably an electron 
inflow to the reactive bonds up to a point of saturation at the transition state (TS); such an inflow stabilizes the TS. The second is the idea that a longer bond stretches more up to the TS and this implies a further rise for the reaction barriers. The third idea is that the intrinsic barrier can increase with the absolute value of exothermicity and endothermicity of the reactions; when the dynamic parameter of $\operatorname{ISM}(\Lambda)$ verifies the condition $\Lambda>>\left|\Delta E^{0}\right|$ the intrinsic barrier is independent of $\left|\Delta E^{0}\right|$ and ISM leads to the quadratic equation of Marcus as a particular case. For atom, proton and methyl transfers it was possible to deconstruct the reactivity of many molecular systems into its determining factors, and to reconstruct them to examine the success and failure of widely used structure-reactivity relationships (SRR). ${ }^{1,2}$

The characterization of the nature of bonding in the transition states is an important topic for the rationalization of several organic processes and for the design of novel electrocyclization syntheses. ${ }^{3,4}$ Since ISM is presently the most complete formalism for SRR, this approach will be applied to the case of pericyclic and pseudopericyclic reactions. It will be shown that ISM can complement quantum-mechanical computations in order to provide a physical perception of chemistry as the science that describes matter from the point of view of atomic and molecular properties. From such an analysis new questions arise which can be subsequently addressed through further quantum-mechanical calculations. This will be the methodology employed in the present paper.

\section{Pericyclic and Pseudopericyclic Reactions}

Pericyclic reactions were originally defined by Woodward and Hoffmann ${ }^{5}$ as 'reactions in which all first-order changes in bonding relationships take place in concert as a closed curve'. Subsequently, those authors subdivided pericyclic transformations into five categories: cycloadditions, electrocyclic, sigmatropic, cheletropic and group-transfer reactions. Relevant for the present application is the cheletropic class, reactions in which two bonds are made or broken to a single atom.

In pseudopericyclic reactions, although there is a concerted electronic rearrangement through a cyclic array of atoms, the participation of non-bonding orbitals, orthogonal to the bonding ones, leads to one or more orbital disconnections because the orbitals of the closed loop meet at a single atom but do not overlap. ${ }^{6}$ By contrast, pericyclic reactions do not experience such disconnections and have a strong aromatic character.

Although there have been numerous electronic structure calculations for pericyclic and pseudo-pericyclic (see ref. 7 and references therein; also refs. 8, 9 in this journal) none has specifically focused on the correlation between bond order and geometric parameters.

Birney and coworkers ${ }^{4}$ carried out $a b$ initio molecular-orbital theory calculations (MPF2(FC)/6-31G* optimized geometries and MP4/D95**+ZEP single point energies) to investigate the number and type of disconnections, in the loop of interacting orbitals, necessary to obtain the 'energetic benefits' of pseudopericyclic in decarbonylation reactions. Several cycloketones, namely 3-cyclopentenone and derivatives, were examined and classified according to the number of orbital disconnections (Scheme 1). 
Pericyclic reactions (cyclic orbital overlap)

A<smiles>O=C1CC=CC1</smiles>

1

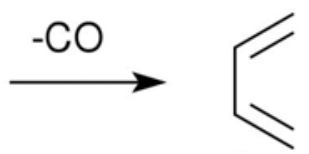

2
B

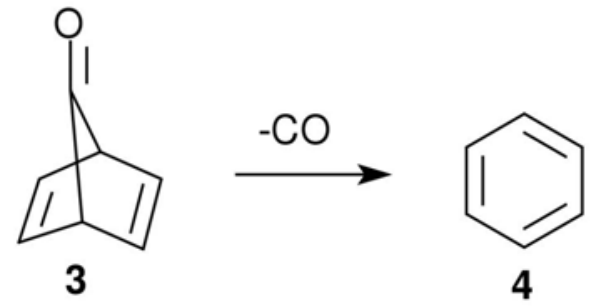

Pseudopericyclic reactions (two orbital disconnections)

C<smiles>O=C1C=COC1=O</smiles>

5

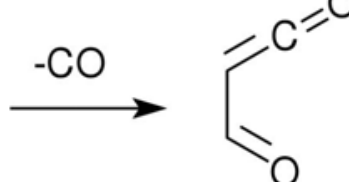

6<smiles>O=C1C=CNC1=O</smiles>

7

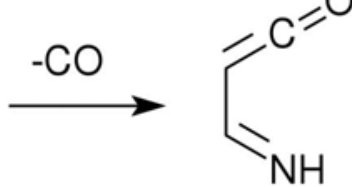

8

One orbital disconnection

E<smiles>O=C1CC1</smiles>

9

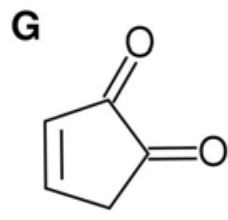

13

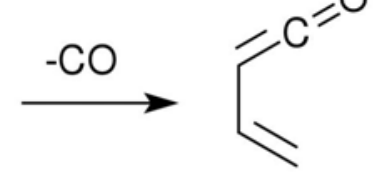

14
$\mathbf{F}$<smiles>O=C1CC=CO1</smiles>

11

H

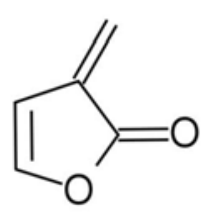

15

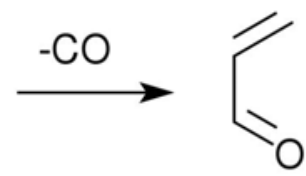

12

\section{Scheme 1}

Two thermal decarbonylations were considered to highlight the differences between the topology of pericyclic and pseudopericyclic reactions, as illustrated in Scheme 2. Decarbonylation of cyclopentenone $\mathbf{1}$ is a typical allowed pericyclic-reaction, because there is a cyclic orbital-overlap around the ring of interacting atoms. The requirement for orbital overlap at the transition state was predicted by Woodward and Hoffmann ${ }^{5,10}$ to result in the departure of carbon monoxide out of the butadiene plane via a disrotatory pathway. The transition state is not planar, as a direct consequence of the need for cyclic orbital-overlap. This is general for hydrocarbon pericyclic reactions.

In contrast, for the decarbonylation of furandione $\mathbf{5}$ one finds two atoms where two orthogonal sets of orbitals meet, but do not overlap. There is an orbital disconnection at a cumulene carbon and the other at an atom (oxygen) with lone pairs. Because no electrons are exchanged between the in-plane and out-of-plane orbitals, the transition state for decarbonylation 
of 5 is orbital-symmetry allowed when the $\mathrm{CO}$ departs in the plane of the molecule. Therefore, the transition state for decarbonylation of furandione is planar (pseudopericyclic reaction) whereas that for cyclopentenone is nonplanar (pericyclic reaction). However, crowding at the transition state can lead to small distortions from planarity. Reactions [4+2] of formylketene and imidoylketene have steric crowding that leads to nonplanar distortions. Cheletropic decarbonylations of furandione and derivatives were chosen by Birney et al. because they have less crowding, so that distortions from planarity should reflect electronic factors rather than steric ones. $^{11}$

Birney et $a .^{4}$ have calculated the energy barriers, $\Delta E^{\ddagger}$, and the reaction energy, $\Delta E^{0}$, for all the reactions presented in Scheme 1 and have tried to examine the similarity of the different reactions in terms of the Bell-Evans-Polanyi relationship (BEPR). The main conclusions provided by these authors are that two orbital-disconnections are sufficient to allow a reaction to be pseudopericyclic and to have an approximately planar structure for the transition state. A single disconnection may, but will not always lead to a nearly planar transition-state structure. With respect to cyclopropanone Birney and coworkers ${ }^{4}$ stated that although formally orbital symmetry-allowed, because it possess a high reaction energy-barrier and lacks an energetic benefit of concert, it should be considered to be 'effectively forbidden'.

The methodology employed was essentially correct, because quantum-mechanical calculations have difficulty in unravelling trends of reactivity within families of reactions. Although extremely useful, those calculations need to be complemented by simple models capable of such unravelling and providing an overview on SRR. However, BEPR is not sufficiently powerful to suggest the expected electronic trends, because it only takes into account the thermodynamic contribution. This difficulty suggests two questions. Is there any criterion to measure the pericyclic and pseudopericyclic electronic-character of the decarbonylation reactions? If such a criterion can be found, is there any geometric characteristic of the transitionstate structure, calculated by $a b$ initio methods, which correlates with such a quantitative criterion? A criterion of electronic nature, free from the effect of $\Delta E^{0}$ as in the model of BEPR, but also free from the effect of force constants and bond lengths, is the transition-state bondorder, $n$. Thus, ISM can be used as a complement to those $a b$ initio calculations, instead of the classical BEPR.

Furthermore, the importance of $n^{\ddagger}$ for chemical reactivity is evidence based, when the transition-state bond-order is expressed in terms of the electrophilicity index of Parr. ${ }^{12}$ Barroso et al. ${ }^{13}$ have employed Partial Least Squares to assess parameters related to chemical kinetics. It is shown that activation energies for the set of atom-transfer reactions can be mostly described in terms of the reaction energy, $\Delta E^{0}$, and Parr's electrophilicity index, reinforcing the view that $n^{\ddagger}$ is an indispensable molecular parameter for any SRR.

\section{Transition-state Bond-orders}

For a prototype bond-breaking-bond-forming reaction $\mathrm{A}+\mathrm{BC} \rightarrow \mathrm{AB}+\mathrm{C}, \mathrm{ISM}$ at the harmonic approximation can be simply applied with the following formalism 


$$
\frac{1}{2} f_{r} x^{2}=\frac{1}{2} f_{p}(d-x)^{2}+\Delta E^{0}
$$

Equation (1) allows one to estimate the bond extension, $x$, of the reactant B-C bond up to the transition state, which is related to the energy barrier $\Delta E^{\ddagger}$ of the reaction,

$$
\Delta E^{*}=\frac{1}{2} f_{r} x^{2}
$$

as long as the sum of the reactant and product bond extensions to the transition state, $d$, is known. ISM provides such a value

$$
d=\left\{\frac{a^{\prime} \ln 2}{n^{\ddagger}}+\frac{a^{\prime}}{2}\left[\frac{\Delta E^{0}}{\Lambda}\right]^{2}\right\}\left(l_{r}+l_{p}\right)
$$

In Equation (3) $a^{\prime}$ is a constant $\left(a^{\prime}=0.156\right)$ and $\Lambda$ is a coupling parameter which reflects how the reaction energy is accumulated in the internal reactive modes.

For the pericyclic and pseudopericyclic reactions under study, the reaction barrier is assessed in terms of the prototype triatomic model, where $n^{\ddagger}=0.5$ when bond order is conserved along the reaction coordinate,

$$
n_{B C}+n_{A B}=1
$$

The nonplanar nature of the transition-state for pericyclic reactions is expected to lead to the typical $n^{ \pm}=0.5$. Accordingly, for a decarbonylation reaction such as that of 3 -cyclopentenone, the valence structure of the transition state can be represented as illustrated, in Scheme 2.
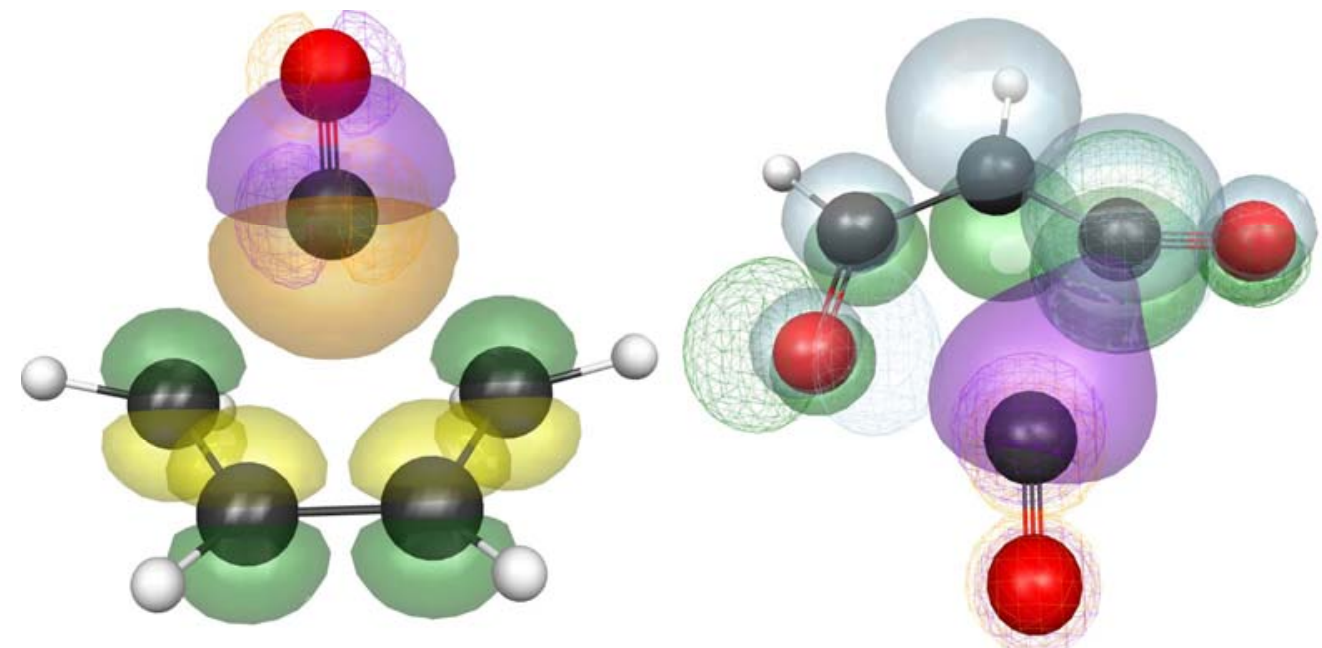

Scheme 2. Orbital interactions in the pericyclic decarbonylation of 1TS. The CO is above the butadiene plane. There is cyclic orbital overlap around the ring of interacting atoms (left panel). Orbital interactions in the corresponding process for the pseudopericyclic 5TS (right panel). (Adapted from ref. 4) 
However, for a planar transition-state the interaction of the $p_{x}$-orbitals of the carbon atoms of butadiene with the $p_{x}$-orbital of the O-atom of the carbonyl group via one of the $\pi$-orbital $\left(\pi_{x}\right)$ of the same group, can delocalize 3-electrons around the carbon and oxygen skeleton as also illustrated in Scheme 2.

For reaction $\mathbf{5} \rightarrow \mathbf{6}$, one has 6 bonds in the reactants and 4 bonds in the products; the TS can be considered, in simple terms, to have an intermediate value, 5 bonds. The expected increase in the bond order of the reactive bonds at the transition state, due to such a delocalization effect of three electrons equally shared by five bonds, is $\Delta n^{\ddagger}=3 /(2 \times 5)=0.3$. So for a pseudopericyclic reaction one expects $n^{\sharp}=0.8$.

Following the procedure employed for sigmatropic shifts and cycloaddition reactions, ${ }^{14}$ for the processes presented in Scheme 1, one can also define a common force-constant of the cyclic ring of five or six members. This force constant is taken as the average of the corresponding stretches of the chemical bonds of the ring. For example, for compound 3 , one has $f_{\mathrm{eff}}=\left(2 f_{\mathrm{C}=\mathrm{C}}+\right.$ $\left.4 f_{\mathrm{C}-\mathrm{C}}\right) / 6$ and for compound 5 is $f_{\mathrm{eff}}=\left(f_{\mathrm{C}=\mathrm{C}}+2 f_{\mathrm{C}-\mathrm{C}}+2 f_{\mathrm{C}-\mathrm{O}}\right) / 5$. The same is valid for the bond lengths, $l_{\mathrm{r}}+l_{\mathrm{p}}=2 \mathrm{x}\left(2 l_{\mathrm{C}=\mathrm{C}^{+}}+4 l_{\mathrm{C}-\mathrm{C}}\right) / 6$ and $l_{\mathrm{r}}+l_{\mathrm{p}}=2 \mathrm{x}\left(l_{\mathrm{C}=\mathrm{C}}+2 l_{\mathrm{C}-\mathrm{C}}+2 l_{\mathrm{C}-\mathrm{O}}\right) / 5$ respectively. This procedure is not particularly critical, because the effects of $f$ and $l$ tend to compensate; for typical chemical bonds, a shorter bond corresponds to a higher force constant and a longer bond corresponds to a lower force constant.

An immediate consequence of such a definition of reaction coordinate is that the cyclic ring is expanded, but the $\mathrm{CO}$ bond is not involved in this concerted nuclear motion. Therefore, the carbon atom of the CO-group is compressed against the oxygen atom, shortening the $\mathrm{CO}$ distance in the transition state. This implies that the $\mathrm{CO}$ bond-length in the transition state will be closer to the carbon monoxide bond-length $\left(l_{\mathrm{C}=0}=1.13 \AA\right.$ to be compared with $\mathrm{l}_{\mathrm{C}=0}=1.215 \AA$ of $\mathrm{C}=\mathrm{O}$ ). In consequence, the nascent carbon monoxide will be released with a small vibrationalenergy content, as has been experimentally observed. ${ }^{15}$ Under those circumstances, one can safely assume that the dynamic effects encompassed by ISM can be neglected, i. e., $\Lambda>>\left|\Delta E^{0}\right|^{16}$ As previously stated, such a condition corresponds to the particular case of ISM leading to the quadratic equation of Marcus.

Table 1 compiles the $a b$ initio reaction energy-barriers and reaction energies estimated by Birney et al. ${ }^{4}$ Those values were subsequently employed to estimate $n^{\ddagger}$, which fits these $a b$ initio data with the other structural parameters also presented in the same Table.

Cyclopropanone 9 constitutes an exception to the concerted criterion for the estimation of the effective force-constant for the decarbonylation reaction. The three-member ring has too much strain to behave as a carbon-skeleton ring. In fact, Birney et al. ${ }^{4}$ have calculated a very asynchronous pathway; in the transition state one of the breaking bonds is $0.61 \AA$ longer than the other reactive one. The departing CO remains in the molecular plane, but the pathway is nonlinear in that the $\mathrm{CO}$ bends away from the $\mathrm{C}_{2}$ axis of $\mathbf{9}$. Therefore, for this reaction we will consider that the carbon-carbon bonds act as local modes, rather than normal modes acting in a concerted fashion, so the effective force constant is $\left.f_{\text {eff }}=\sqrt{\left(f_{1}^{2}\right.}+f_{2}^{2}\right)$. In a three-member ring two bonds are sufficient to define the ring geometry; thus $f_{\text {eff }}=\sqrt{ } 2 f_{\mathrm{C}-\mathrm{C}}$. 
Table 1. Energy barriers, $\Delta E^{\ddagger}$, reaction energies, $\Delta E^{0}$, and transition-state bond-orders, $n^{\ddagger}$, for decarbonylations reactions of Scheme $1^{\mathrm{a}}$

\begin{tabular}{ccccccccc}
\hline reagent & $\Delta E^{\ddagger \mathrm{b}}$ & $\Delta E^{0 \mathrm{~b}}$ & $f / 10^{3}$ & $l_{\mathrm{r}}+l_{\mathrm{p}}$ & $d$ & $n^{\ddagger}$ & $\mathrm{O}_{1}-\mathrm{C}_{2}-\mathrm{C}_{6}{ }^{\mathrm{b}}$ & $\begin{array}{c}\mathrm{n}^{\mathrm{o}} \text { orbital } \\
\text { disconnections }\end{array}$ \\
\hline 1 & 204.8 & 79 & 3.3 & 2.99 & 0.630 & 0.51 & 132.5 & 0 \\
3 & 63.5 & -135.9 & 3.7 & 2.94 & 0.514 & 0.62 & 140.4 & 0 \\
5 & 80.2 & 18.8 & 3.43 & 2.96 & 0.405 & 0.79 & 153.3 & 2 \\
7 & 144.2 & 79.4 & 3.4 & 2.94 & 0.4865 & 0.65 & 150.9 & 2 \\
$9^{\mathrm{c}}$ & 156.8 & -93.6 & 3.8 & 3.074 & 0.650 & 0.51 & - & 1 \\
11 & 203.2 & 55.6 & 3.43 & 2.96 & 0.638 & 0.50 & 135.6 & 1 \\
13 & 156.3 & 73.6 & 3.3 & 2.99 & 0.532 & 0.61 & 140.8 & 1 \\
15 & 224.5 & 122 & 3.43 & 2.96 & 0.609 & 0.525 & 136.4 & 1 \\
\hline
\end{tabular}

${ }^{\text {a }}$ Energies in $\mathrm{kJ} / \mathrm{mol}$, force constants in $\mathrm{kJ} \mathrm{mol}^{-1} \AA^{-2}$, bond lengths in $\AA$, angles in degrees.

${ }^{\mathrm{b}}$ Ref. $4{ }^{\mathrm{c}}$ force constant for two local modes.

Data employed in the calculations: $f_{\mathrm{C}-\mathrm{C}}=2.7 \times 10^{3}, l_{\mathrm{C}-\mathrm{C}}=1.537 ; f_{\mathrm{C}-\mathrm{N}}=2.95 \times 10^{3}, l_{\mathrm{C}-\mathrm{N}}=1.472$; $f_{\mathrm{C}=\mathrm{C}}=5.77 \times 10^{3}, l_{\mathrm{C}=\mathrm{C}}=1.335 ; f_{\mathrm{C}-\mathrm{O}}=3 \times 10^{3}, l_{\mathrm{C}-\mathrm{O}}=1.497 .^{17}$

According to the $n^{*}$ values presented in Table 1, the decarbonylation reactions (Scheme I) can be ordered from the more pure pericyclic to the extreme of pseudopericyclic reactions (indicated through the relevant reactant),

$$
\begin{gathered}
\mathbf{1}=\mathbf{9}=\mathbf{1 1} \approx \mathbf{1 5}<\mathbf{1 3} \approx \mathbf{3} \leq \mathbf{7}<\begin{array}{c}
\mathbf{p} \\
\text { pericyclic } \\
\left(n^{\ddagger}=0.5\right)
\end{array} \\
\text { pseudopericyclic } \\
\left(n^{\ddagger}=0.8\right)
\end{gathered}
$$

Pericyclic reactions include a zero orbital-disconnection reaction for compound $\mathbf{1}$, but also reactions with one orbital-disconnection 9, 11 and 15. Cyclopropane 9 decarbonylation was considered by Birney et $a l^{4}$ to be a forbidden reaction, but a proper definition of the reaction coordinate allows one to place this reaction as an allowed pericyclic-reaction as predicted by Woodward and Hoffmann. Reactions with zero orbital-disconnection $\mathbf{3}$ and one orbitaldisconnection 13 are intermediate cases. The same can be said about 7 which has two orbitaldisconnections. Only furandione $\mathbf{5}$ with two orbital-disconnections is a clear example of a pseudopericyclic reaction with $n^{\ddagger}=0.79$, very close to the predicted value of $n^{\ddagger}=0.80$.

Associated with the number of orbital disconnections is the geometry of the transition state. Birney et al. ${ }^{4}$ have stated that the nearly linear $\mathrm{O}(1) \mathrm{C}(2) \mathrm{C}(6)$ geometry $\left(\theta_{1,2,6}=153.3^{\circ}\right)$ in 5 suggests that it is more appropriate to consider the reverse reaction, the interaction of carbon monoxide with formylketene 6, in which donation of the CO lone-pair to the in-plane ketene HOMO is the dominant interaction, and leads to the nearly linear geometry. In fact, of all the geometrical parameters estimated for the transition state by Birney and coworkers, the one that 
correlates with $n^{\ddagger}$ is the angle $\mathrm{O}(1) \mathrm{C}(2) \mathrm{C}(6)$. A good linear correlation is found between $\theta_{1,2,6}$ and $n^{\star}$ as illustrated in Figure 1. In fact, for a true orbital disconnection no electrons are exchanged between the in-plane and out-plane orbitals. But such orthogonally can be broken and provide a source of an increase in $n^{*}$ as long as there is a partial orbital-overlap at a single atom, and this can be controlled by the $\mathrm{O}(1) \mathrm{C}(2) \mathrm{C}(6)$ angle.

The only nitrogen derivative studied, 2,3-pyrroledione 7, deviates from the correlation, but that might be a consequence of the rehybridization of the $\mathrm{N}$-atom at the 3-position from $s p^{2}$ towards $s p^{3}$ to accommodate the calculated increase in negative charge at this nitrogen atom as suggested by Birney et al. ${ }^{4}$

An alternative interpretation can be provided on the basis of the electron inflow to the reactive bonds up to a point of saturation at TS. As Arnaut et al. have shown, ${ }^{2 b}$ for the prototype reaction, the overall bond order at the TS

$$
m=2 n^{*}
$$

can be measured by the electrophilicity index of Parr ${ }^{12}$

$$
m=\frac{-\mu_{e l}}{\eta_{e l}}=\frac{I_{P}+E_{A}}{I_{P}-E_{A}}
$$

and through such a procedure absolute rates of elementary reactions have been calculated. ${ }^{1,2,18}$ In Equation (6) $\mu_{\mathrm{el}}$ represents the chemical potential, $\eta_{\mathrm{el}}$ the chemical hardness and the $I_{\mathrm{P}}$ and $E_{\mathrm{A}}$ represent the ionization potential and electroaffinity of the reactive molecules for the pair $I_{\mathrm{P}}(\mathrm{BC})$ and $E_{\mathrm{A}}(\mathrm{AB})$ or $I_{\mathrm{P}}(\mathrm{AB})$ and $E_{\mathrm{A}}(\mathrm{BC})$.

Equation (6) deals with the electronic stabilization when $\mathrm{A}, \mathrm{B}$ and $\mathrm{C}$ interact at the transition state, $\{\text { A...B...C }\}^{*}$. The electrophilicity index $m$ proposed by Parr represents the saturation point for electron inflow as the ratio between the negative of the electronic chemical potential and the chemical hardness, and is a good measure for the extra electronic stabilization of TS. In general, the point of electron saturation at TS is attained for the pair which provides the highest $m$ value.

In fact, Parr and coworkers have explored variational principles in order to understand chemical reactivity on the basis of the changes in electron density associated with a chemical reaction. They concluded ${ }^{12,19}$ that the abundance of chemical knowledge underscores the need for incisive and inclusive tools for systematizing chemical data. Along those lines and based on insights provided by DFT studies, those authors proposed the indices above presented for the elucidation of chemical reactions. As stated, Barroso et al. ${ }^{13}$ have employed Partial Least Squares to assess parameters related to chemical kinetics, including the electrophilicity index of Parr, with the same goal.

Thus, the nitrogen compound 7 can be compared with the oxygen compounds (5) by taking into account the influence of the electrophilicity index of Parr. From ref. 2a (Table 6.4) one realizes that $m$ is higher for reactions involving $\mathrm{O}$-atoms, $m\left(\mathrm{OH}+\mathrm{H}_{2} \rightarrow\right)=1.327$, than for those 
with $\mathrm{N}$-atoms, $m\left(\mathrm{NH}_{2}+\mathrm{H}_{2} \rightarrow\right)=1.154$. This suggests that the decarbonylation reaction (D) should be $n^{\ddagger}$ deficient with respect to the reaction 5 by $\Delta n^{\ddagger}=(1.154-1.327) / 2=0.0865$.

Figure 1 gathers bond-orders calculated using ISM. It is seen that a linear trend is clearly followed. After introduction of the correction, reaction (D) is also included in the common trend.

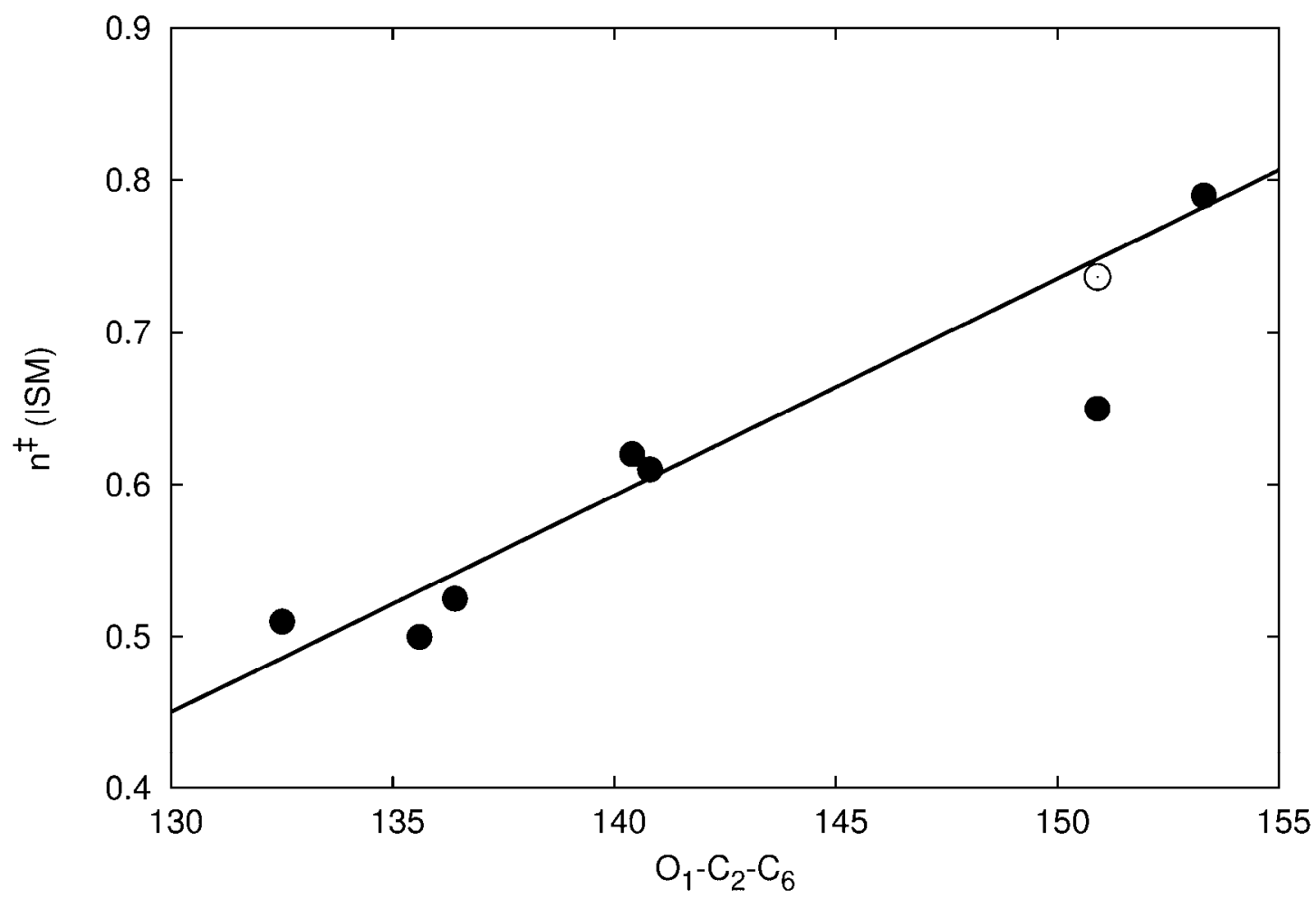

Figure 1. Bond-order, as extracted from Equations (1-3), for the set of reactions under study. The open circle corresponds to the corrected value for reaction (D), Equations (5) and (6). The solid line is a linear fit to the values for the seven reactions, including the above, corrected, point.

To further access the validity/reliability of this simple model, some electronic structure calculations were performed. We chose three systems, encompassing the three types of reactions (A, B and C). For each system, the transition state structure was determined using the 6$311 \mathrm{G}(\mathrm{d}, \mathrm{p})^{20}$ basis set at the Hartree-Fock level, resorting to the GAMESS-US ${ }^{21}$ code and using both the restricted and unrestricted approaches. These results produced the transition-state geometries depicted in Figure 3. The nature of the stationary point was assessed by the calculation of the Hessian matrix and inspection of the eigenvalues. For each reaction, the Hessian possessed only one negative eigenvalue, as expected for a transition state. At the transition state geometry, a Natural Bond $\operatorname{Order}^{22}$ analysis was performed, and the values obtained are shown in Figure 2. Note that for reaction $(\mathbf{C})$, the calculated bond order refers to the angle $\mathrm{O}-\mathrm{C}-\mathrm{C}$, which is taken as the reaction coordinate. As expected, there is little difference 
between the Restricted Hartree- Fock (RHF) and Unrestricted Hartree-Fock (UHF) bond-order values.

Furthermore, the trend predicted by the simple approach resorting to the ISM model (cf. Figure 1) is closely followed.

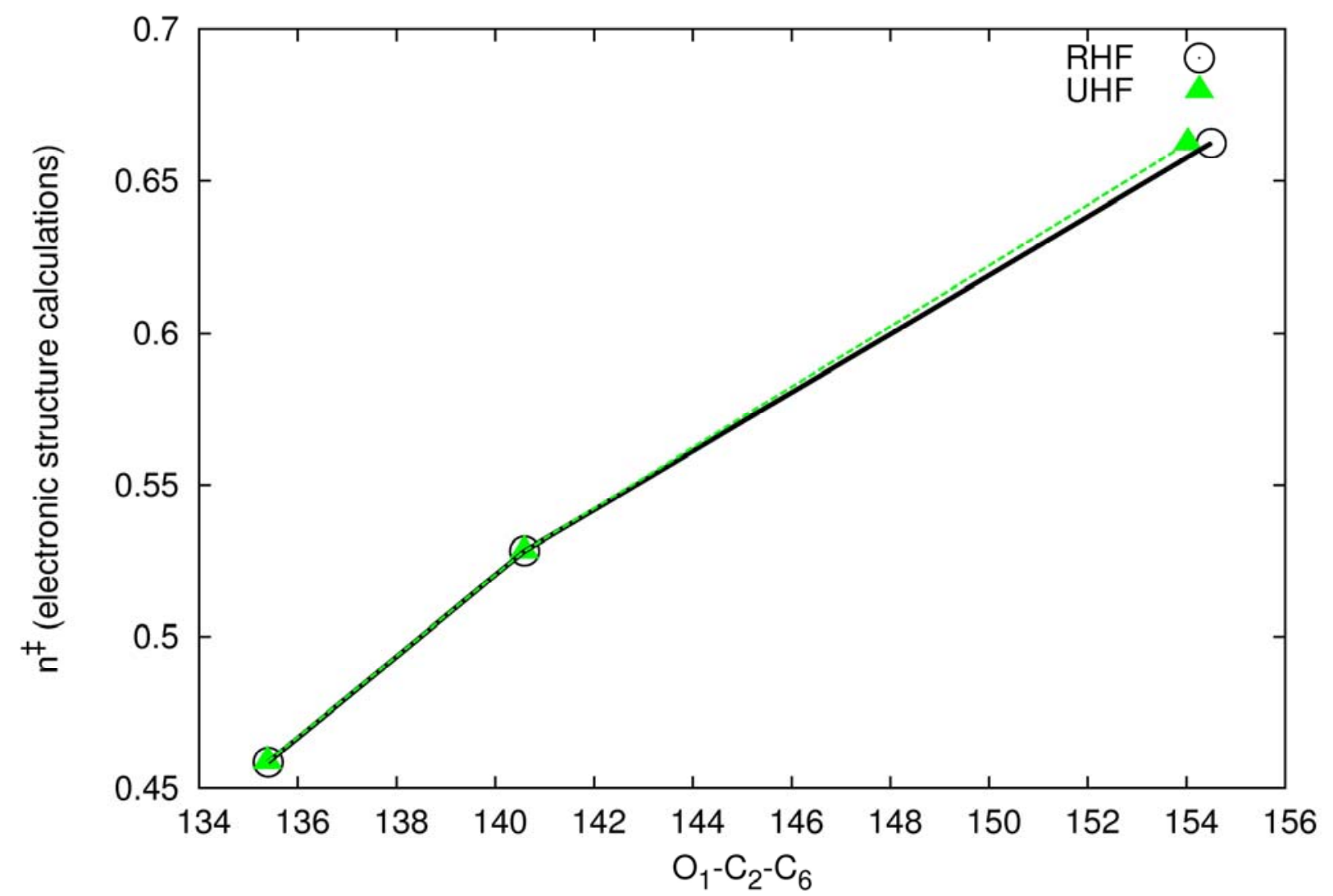

Figure 2. Same as Figure 1, using electronic structure calculations at the Hartree-Fock level for the selected reactions (see text). 

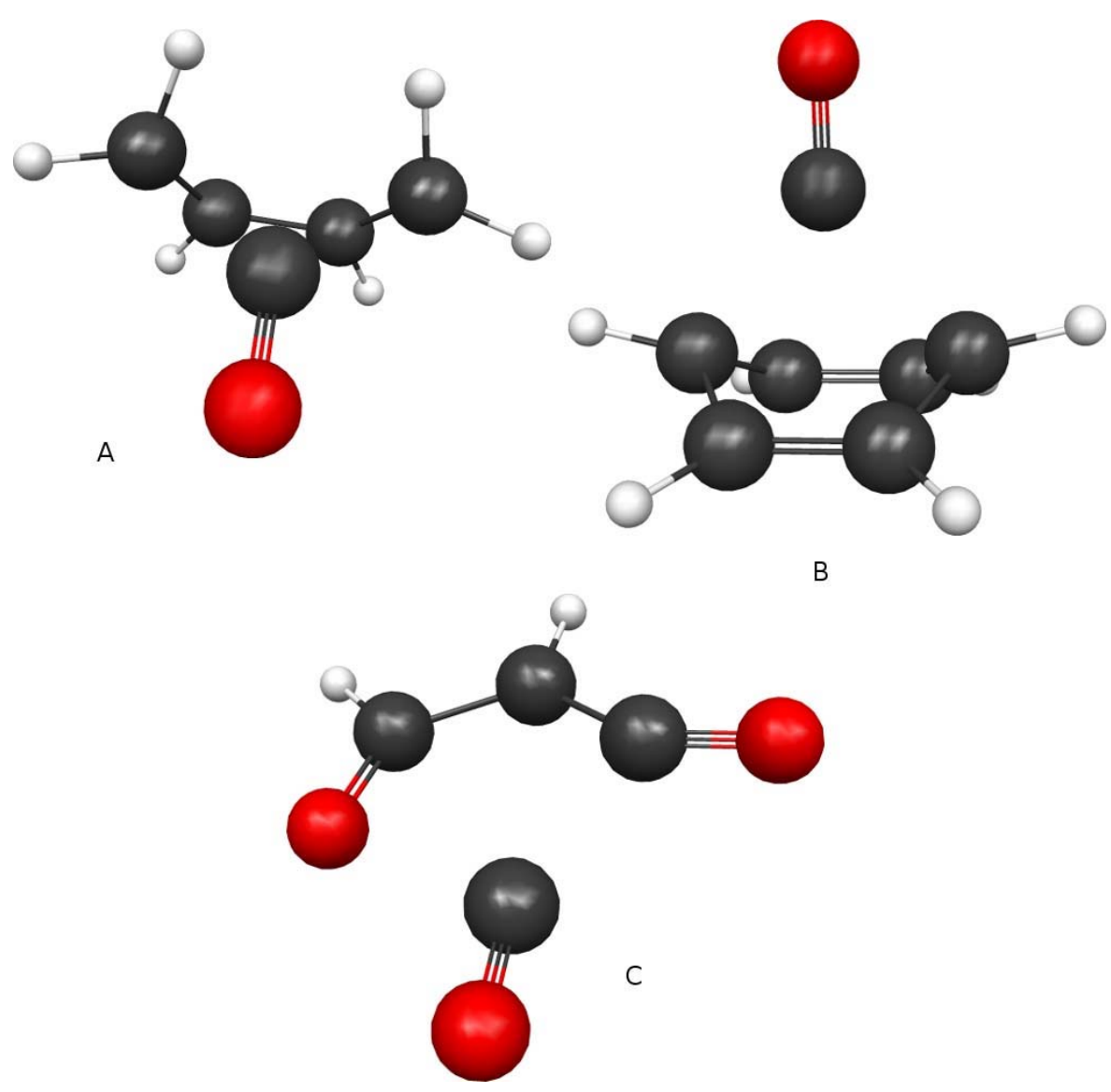

Figure 3. Ball-and-stick representation of the transition-state geometries determined in this work, at the Hartree-Fock level. Labels as in Scheme 1.

\section{Concluding Remarks}

ISM is presently the most complete SRR available in the literature. In spite of its simplicity, even for complex systems as those of cheletropic decarbonylation reactions presented here, it provides results in good agreement with the more time consuming quantum mechanical calculations. Furthermore, it can provide a broader view of chemical reactivity within families of reactions.

\section{Acknowledgements}

The authors gratefully acknowledge Fundação para a Ciência e Tecnologia, FCT, Portugal, for financial support through project PTDC/QUI/70637/2006.

\section{References}

1. Arnaut, L. G.; Formosinho, S. J. Chem. Eur. J. 2008, 14, 6578. 
2. (a) Arnaut, L. G.; Formosinho, S. J.; Burrows, H. D. Chemical Kinetics. From Molecular Structure to Chemical Reactivity; Elsevier:Amsterdam, 2007; Chaps. 6, 7, 11, 13. (b) Arnaut, L. G.; Pais, A. A. C. C.; Formosinho, S. J.; Barroso, M. J. Am. Chem. Soc. 2003, 125, 5236.

3. (a) Rodríguez-Otero, J.; Cabaleiro-Lago, E. M. Angew. Chem. Int. Ed. 2002, 41, 1147. (b) Alajarín, M.; Ortin, M. M.; Sánchez-Andrada, P.; Vidal, A. J. Org. Chem. 2006, 71, 8126.

4. Birney, D. M.; Ham, S.; Unruch, G. R. J. Am. Chem. Soc. 1997, 119, 4509.

5. Woodward, R. B.; Hoffmann, R. Angew. Chem., Int. Ed. Engl. 1969, 8, 781.

6. Ross, J. A.; Seiders, R. P.; Lemal, D. M. J. Am. Chem. Soc. 1976, 98, 4325.

7. Bachrach, S. M. Computational Organic Chemistry, Wiley: Hoboken, N. J., 2008; Chap. 3.

8. Gajewski, J. J.; Conrad N. D.; Emrani, J; Gilbert, K. E. Arkivoc 2002, (iv), 18.

9. Silva, M. A.; Pellegrinet, S. C.; Goodman, J. M. Arkivoc 2003, (x), 556.

10. Woodward, R. B.; Hoffmann, R. The Conservation of Orbital Symmetry; Verlag Chemie, GmbH: Weinheim, 1970.

11. (a) Birney, D. M. J. Org. Chem. 1994, 59, 2557. (b) Ham, S.; Birney, D. M. J. Org. Chem. 1996, 61, 3962.

12. (a) Parr, R. G.; Szentpály, L. V.; Liu, S. J. Am. Chem. Soc. 1999, 121, 1922. (b) Ayers, P. W.; Parr, R. G. J. Am. Chem. Soc. 2001, 123, 2007.

13. Barroso, M.; Pereira, J. C.; Pais, A. A. C. C.; Arnaut, L. G.; Formosinho, S. J. Mol. Phys. 2006, 104, 731.

14. Formosinho, S. J. Tetrahedron 1986, 42, 4557.

15. (a) Jimenez, R.; Kable, S. H.; Loison, J.-C.; Simpson, C. J. S. M.; Adam, W.; Houston, P. L. J. Phys. Chem. 1992, 96, 4188. (b) Prather, K. A.; Rosenfeld, R. N. J. Phys. Chem. 1991, 95, 6544.

16. Formosinho, S. J. In Theoretical and Computational Models for Organic Chemistry; Formosinho, S. J.; Csizmadia, I. G.; Arnaut, L. G. Eds.; NATO ASI Series, Kluwer: Dordrecht 1991; Vol. 339, p 159.

17. Gordon, A. J.; Ford, R. A. The Chemist's Companion, John Wiley:New York, 1972, pp 107 and 114.

18. Barroso, M.; Arnaut, L. G.; Formosinho, S. J. Chem. Phys. Chem 2005, 6, 363. (b) Barroso, M.; Arnaut, L. G.; Formosinho, S. J. J. Phys. Chem. A 2007, 111, 591.

19. Ayers, P. W.; Parr, R. G. J. Am. Chem. Soc. 2000, 122, 2010.

20. Krishnan, R.; Binkley, J. S.; Seeger, R.; Pople, J. A. J. Chem. Phys.1980, 72, 650. 
21. Schmidt, M. W.; Baldridge, K. K.; Boatz, J. A.; Elbert, S. T.; Gordon, M. S. Jensen, J. H.; Koseki, S.; Matsunaga, N.; Nguyen, K. A.; Su, S.; Windus, T. L.; Dupuis, M.; Montgomery, J. A. J. Comput. Chem. 1993, 14, 1347.

22. Glendening, E. D.; Badenhoop, J. K.; Reed, A. E.; Carpenter, J. E.; Bohmann, J. A.; Morales, C. M.; Weinhold, F. NBO 5.G.,Theoretical Chemistry Institute, University of Wisconsin, Madison, WI, 2001; http://www.chem.wisc.edu/ nbo5. 